\title{
Chyle Leakage and Early Enteral Feeding following Pancreatico-Duodenectomy: Management Options
}

\author{
H.Z. Malik J. Crozier L. Murray R. Carter \\ West of Scotland Pancreatic Unit, Glasgow Royal Infirmary, Glasgow, UK
}

Key Words

Chyle leak · Early enteral feeding $\cdot$ Pancreatic resection

\begin{abstract}
Background: Chyle leaks are a rare complication following abdominal surgery. The aim of this study is to describe the management of chyle leaks following surgery for pancreatico-duodenal malignancy. Methods: Data were collated from a consecutive series of 105 patients who had undergone a pancreatico-duodenectomy for malignancy. From this cohort, patients who developed significant chyle leaks, defined as drainage of more than $600 \mathrm{ml}$ of amylase-poor chylous fluid per day, were identified and their management reviewed. Results: A total of 7 (6.7\%) patients with significant chyle leaks were identified. All but one of the chyle leaks were identified between the 5 th and 9th post-operative day. Early restoration of enteral feeding appeared to increase the incidence of chyle leak. Six of the 7 patients were successfully treated conservatively with total parenteral nutrition and after a median of 7.5 days the chyle leak had resolved. One patient required a peritoneovenous shunt for chylous ascites. Conclusion: This study has described a high incidence of chyle leak among patients undergoing surgery for pancreatico-duodenal malignancy. Early introduction of enteral feed may encourage development. However, patients who develop an abdominal chyle leak remain clinically well and the leak can be managed relatively easily with parenteral nutrition.

Copyright $\odot 2007$ S. Karger AG, Basel
\end{abstract}

\section{Introduction}

Chylous discharge following abdominal surgery is uncommon and its management is poorly described. Chyle is transported in lymphatics that drain the abdominal and retro-peritoneal viscera and join ascending lymphatic trunks, which drain the lower extremities and the pelvis. As these trunks ascend they form a saccular dilatation called the cisterna chyli. The cisterna chyli lies in front of the first and second lumbar vertebrae and drains into the thoracic duct, which carries the lymph into the thoracic cavity. Disruption of these lymphatic pathways can result in both chylothorax as well as chylous ascites. A variety of mechanisms can result in a chyle leak ranging from congenital defects of the lymphatic system to malignancy or trauma [1-22]. However, chyle leaks are also a recognised complication of abdominal, thoracic and head and neck surgery $[1-2,4-9,12-13,15,18]$.

In the published literature there have been numerous case reports as well as collective reviews of chylothorax [2-21]. However, there are relatively little data on chylous ascites. Leibovitch et al. [22] reviewed the literature on postoperative chylous ascites. The most common surgical cause of chylous ascites was found to be abdominal aortic surgery accounting for $81 \%$ of the cases of chylous ascites reported in the literature. Despite this, less than $1 \%$ of patients undergoing aortic surgery suffer from this complication. Other causes include retroperitoneal dissection for urological and gynaecological malignancy as

Mr. R. Carter

West of Scotland Pancreatic Unit, Glasgow Royal Infirmary

12-14 Alexandra Parade

Glasgow G31 2ER (UK)

Tel. +44 141211 5129, Fax +44 141232 0701, E-Mail rcarter@clinmed.gla.ac.uk 
well as spinal surgery. Hoffman et al. [23] reported a case of chylous ascites following a pancreatico-duodenectomy that was managed by insertion of a peritoneovenous shunt. The main difference between chylothorax and chylous ascites is that chylothorax unlike chylous ascites is invariably associated with an inflammatory response with a high white count and C-reactive protein. Furthermore, as a chylothorax is nearly always due to a disruption of the thoracic duct itself, there is a target for surgical intervention. However, in chylous ascites there is generally not a disruption to one large lymphatic trunk that can be ligated, thus conservative management is usually the main stay in the management of chylous ascites.

A number of techniques have been used in the management of chylous ascites. Aalami et al. [24] reviewed the literature, consisting of 156 patients identified through case reports, and found that in two thirds of cases chylous ascites was managed conservatively. Conservative modalities used in the management of chylous ascites included repeated paracentesis; use of high-protein, low-fat enteral nutrition as well as total parenteral nutrition. Total parenteral nutrition has been shown to be highly effective in the management of chylous ascites with success rates from 60 to $100 \%$ having been reported [25-28]. More recently the use of somatostatin has also been advocated in the management of chylous ascites, although its mechanism of action remains unclear [29-33]. Spiro et al. [34], however, found that among patients with chylothorax the presence of a chylous leak of greater than $600 \mathrm{ml}$ per day was unlikely to resolve with conservative management. In refractory cases surgery has been used to deal with the disruption in the lymphatic flow. Surgical options available include the use of peritoneovenous shunts or the direct ligation of the disrupted lymphatic channels [24].

There is little doubt that enteral nutrition carries advantages over total parenteral nutritional support in that it is easier to administer, more physiologic and less expensive. There may be preservation of gut barrier function and prevent structural alterations induced by starvation and injury. The rationale for feeding the gut early after surgery is that peristalsis of the small intestine recovers 6-8 $\mathrm{h}$ after surgical trauma, and that a moderate absorptive function is preserved even in absence of peristalsis. Moreover, the direct passage of food stuff in the gut lumen increases splanchnic blood flow and stimulates gut immune system. There seems little effect on anastomotic healing and the use of early enteral nutrition has been shown to be well tolerated in the post-operative period in colorectal [35-36] and gynaecological surgery [37]. Following the suggestion that early enteral support could be beneficial in terms of reducing post-operative infectious complications and improving patient's outcome, we modified our peri-operative regimen to allow the early introduction of enteral nutrition after pancreatico-duodenectomy, using a naso-jejunal feeding tube placed distal to the anastomoses intra-operatively. It was during this period of early feeding that chyle leak became problematic. The objective of this study is to review the management of patients identified as having chyle leaks, the diagnosis, intra-operative attempts at prevention and subsequent management strategy prior to resolution.

\section{Patients and Methods}

A consecutive series of 105 patients undergoing resection for pancreatico-duodenal malignancy from January 1999 to January 2005 were reviewed. As part of our unit protocol all post-operative patients had their drain fluid analysed for amylase. The presence of a chyle leak was defined as drainage of $>600 \mathrm{ml}$ of chylous (biochemically contained chylomicrons), amylase/bile-free fluid per day. Patients were identified and their case notes reviewed.

A standard pancreatico-duodenectomy was performed on all patients. Division of the jejunum at the level of the first jejunal arcade was performed with the vessels in the jejunal mesentery being ligated. Subsequent resection of the uncinate and head of pancreas in the peri-vascular plane producing $180^{\circ}$ skeletalisation of the superior mesenteric vein and artery. One patient, however, with a small primary within the pancreatic body required elective superior mesenteric vein resection with primary anastomosis to achieve surgical clearance. Reconstruction was by utilising a single retrocolic jejunal loop to create an end-to-side pancreaticojejunostomy, hepatico-jejunostomy and gastrojejunostomy. In those in whom early feeding was instituted, a naso-jejunal feeding tube was passed intra-operatively and placed beyond the most distal anastomosis, feed starting at $30 \mathrm{ml} / \mathrm{h}$ increasing to full enteral support by $48 \mathrm{~h}$, before being reduced with the establishment of normal diet. The type of feed used was Peptisorb. This is a $1-\mathrm{kcal} / \mathrm{ml}$ semi-elemental oligopeptide feed. It has the lowest fat content of all available enteral feeds and the highest percentage of medium-chain triglycerides as the fat source.

We initially addressed our intra-operative technique to see if these leaks could be prevented. Intra-operative identification of mesenteric lymphatics can be difficult particularly in a fasted patient. In an effort to address this issue intra-operatively, we developed a technique of injecting $50 \mathrm{ml}$ 10\% intralipid (Fresenius Kabi $\mathrm{UK}$ ) into the jejunal lumen, just prior to its division. The lymphatic vessels become opalescent and targeted for ligation (fig. 1). Intra-operative measures used to address these lymphatics have included direct suture ligature; Ultracision (Johnson \& Johnson Medical Ltd.); LigaSure (Tyco Healthcare UK Ltd.); and tissue glue - FloSeal (Baxter Healthcare UK Ltd.); however, none have proved universally successful and chyle leak occurrence appeared unrelated to intra-operative factors.

A SPSS version 7.5 statistics package was used to analyse the data with both the $\chi^{2}$ and t tests being used to analyse statistical differences between those who did and did not develop a chyle leak. 
Fig. 1. Per-operative identification of lymphatic channels in the jejunal mesentery using intra-jejunal intralipid. Grey arrow = Inferior vena cava; white arrow = aorta; black arrow $=$ inferior mesenteric vein.

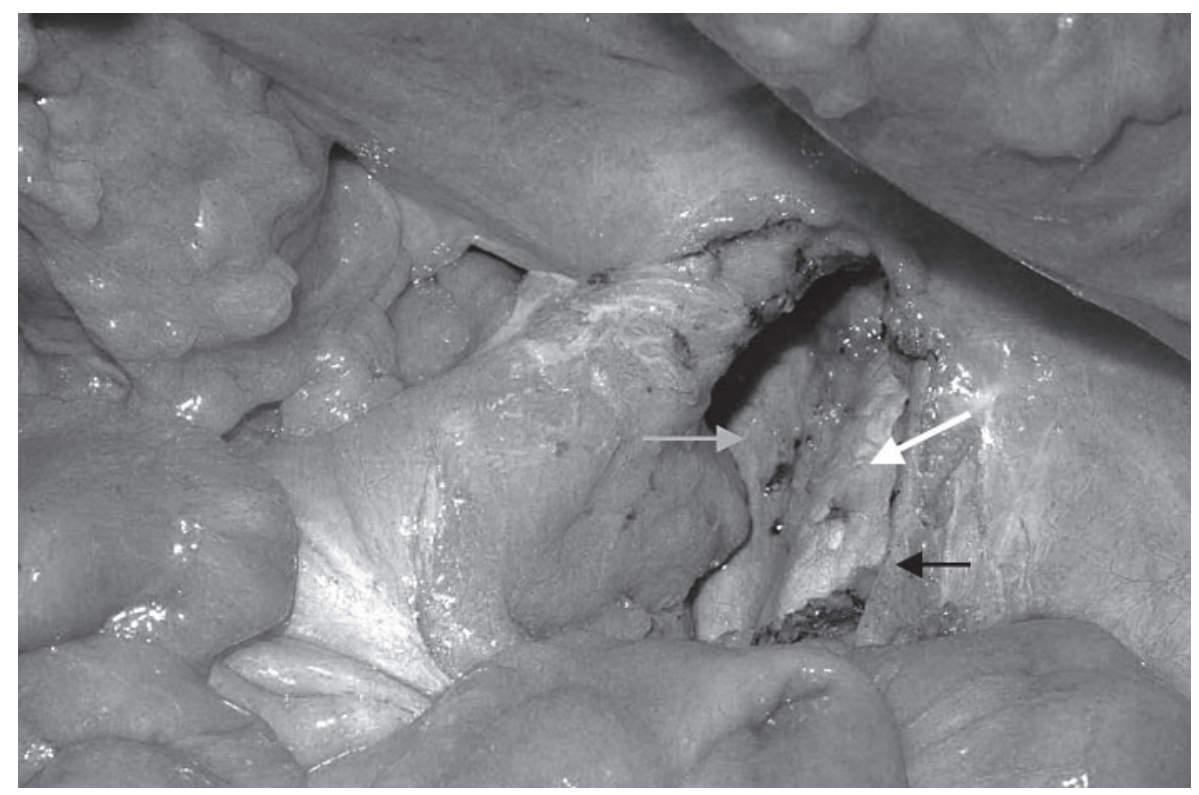

\section{Results}

A total of 7/105 (6.7\%) patients were identified as having a significant chyle leak as defined above. Furthermore, the incidence of pancreatic anastomotic leak was $7 \%$ and the in-hospital mortality rate was $11 \%$ for the whole cohort of patients. Of the 105 patients, 38 underwent insertion of an enteral feeding tube at the time of surgery, with early nutritional support. Among patients who developed a chyle leak, 4 received early enteral nutrition. The clinical features of all patients are represented in table 1 . There was no significant difference in the clinico-pathological features of patients that developed a chyle leak and those who did not (table 1). Of the patients who developed a chyle leak, all except 1 underwent a standard Whipple resection for pancreatic adenocarcinoma. One patient however underwent a pylorus-preserving pancreatico-duodenectomy for a duodenal carcinoma.

The recognition of a chyle leak usually occurred between the 5th and 9th (median 6 days) post-operative day. The maximum volume of chyle drained on a single day ranged from 1,400 to $3,000 \mathrm{ml}$, with the median being $1,900 \mathrm{ml}$. In all cases, the patients remained clinically well with no haemodynamic instability and there was no evidence of an inflammatory response with both the temperature and white blood cell count remaining within normal limits and only a marginal elevation in the C-reactive protein was noted in some of the patients (table 2).
Table 1. Clinico-pathological features

\begin{tabular}{lclc}
\hline $\begin{array}{l}\text { Clinico-pathological } \\
\text { features }\end{array}$ & Chyle leak & $\begin{array}{l}\text { No chyle } \\
\text { leak }\end{array}$ & p value \\
\hline Age* $^{\text {Gender, females }}$ & $61(41-74)$ & $62(45-75)$ & 0.512 \\
T-stage & $5(71 \%)$ & $54(55 \%)$ & 0.429 \\
$\quad$ T1 & 0 & $9(9 \%)$ & 0.630 \\
$\quad$ T2 & $2(29 \%)$ & $32(33 \%)$ & \\
$\quad$ T3 & $5(71 \%)$ & $57(58 \%)$ & \\
N-stage N1 & $3(43 \%)$ & $57(58 \%)$ & 0.398 \\
Early enteral nutrition & $4(57 \%)$ & $34(35 \%)$ & 0.232 \\
\hline
\end{tabular}

* Values represent median and range.

Table 2. Clinical features of patients with chyle leak

\begin{tabular}{lc}
\hline Pattern of chyle leak & \\
\hline Timing of chyle leak, days & $6(5-37)$ \\
Maximum volume, $\mathrm{ml} / 24 \mathrm{~h}$ & $1,900(1,400-3,000)$ \\
Maximum CRP & $28(12-65)$ \\
Maximum WCC & $10(6-14)$ \\
Duration of TPN, days & $7.5(4-32)$
\end{tabular}

Values represent median and range. 
The patient whose chyle leak was managed surgically underwent a classical Whipple resection and was discharged on the 27 th post-operative day. This delayed discharge was due to social factors and after the initial perioperative period this patient remained well in the ward until his discharge date. This patient in the early post-operative period had a persistent high volume drain loss that was low in amylase and lasted 8 days. Due to poor oral intake of fats this remained unrecognised as a chyle leak. The patient was subsequently readmitted 10 days following his discharge with ascites, which proved to be chylous on diagnostic tap. After failure of initial enteral feeding high in medium-chain fatty acids and somatostatin, total parenteral nutrition was instituted, again with a poor clinical response and this patient eventually required a peritoneo-venous shunt.

In all cases initial treatment consisted of conservative management with total parenteral nutrition, and clear oral fluids. Total parenteral nutrition was commenced between 1 and 5 days (median 2 days) after the demonstration of the chyle leak, 5 via a dedicated central venous catheter and one via a peripheral feeding catheter, consisting of a standard 2,500-ml bag containing 2,211 kcal of energy and $13.5 \mathrm{~g}$ of nitrogen per day. Parenteral nutrition was continued until the chyle leak had clinically resolved and the patient had tolerated a period of $24 \mathrm{~h}$ of entral nutrition without recurrence of the leak. Overall, total parenteral nutrition was used for a median of 7.5 days (mean 10.7 days). Among these patients there were no complications related to the delivery of the total parenteral nutrition. Although these patients received somatostatin in the early post-operative period (up to day 5 post-operatively), none of these patients required additional therapy in the form of somatostatin in order to resolve the chyle leak.

\section{Discussion}

Post-operative chyle leaks are a recognised complication of abdominal surgery and have been traditionally difficult to manage with a small proportion of cases necessitating aggressive surgical intervention. Although this complication is thought to be relatively rare following abdominal surgery, we have described an overall incidence of $6.7 \%$ following surgery for pancreatico-duodenal malignancy. This incidence is difficult to explain. Over the period of this study there has been no change in the surgical technique employed, with the jejunal mesenteric vessels being suture ligated. More recently in an at- tempt to control the lymph leak, as identified by the use of intra-operative jejunal injection of intralipid, different techniques were employed including Ultracision and LigaSure as well as tissue glue within a limited number of cases, with little success. Furthermore, all patients underwent a standard pancreatico-duodenectomy without a radical lymphadenectomy. The mechanism of action leading to chyle leak within this group of patients may be due to the lipid content of the enteral feed keeping open the visceral lymphatic channels that have been divided as part of the standard resection thus leading to the persistent chyle leak. Chyle leak did occasionally occur despite a period of gut rest; however, it was during this period of early feeding that chyle leak became problematical, leading to the recognition that the likely source of this chyle was an earlier stimulation of the lymphatic drainage of the small intestine. This trend towards increased rate of chyle leaks amongst patients receiving enteral nutrition did not reach significance. However, as the incidence of chyle leak is relatively rare, this study was not powered to detect such a difference. We have since reverted to a more conservative policy, avoiding enteral intake in the immediate post-operative period, as the use of early nutrition was part of a recent change in our established protocol.

As a result of the demonstrated benefits in colorectal and gynaecological surgery, early restoration of enteral nutrition is being utilised following a wide range of surgical procedures, and overall there seems to be little detriment to introducing nutritional support by this route. A pancreatico-duodenectomy results in interruption of the mesenteric lymphatic plexuses, the hepatico-duodenal ligament and to a lesser degree the retro-peritoneum. The high rate of significant chyle leakage associated with pancreatico-duodenal resection must raise specific questions regarding the use of early re-feeding following pancreatic resection, which appear to be less relevant to other procedures.

When a chyle leak does occur, it appears to be associated with little morbidity. All the patients who developed a chyle leak remained clinically well and unlike chylothorax did not develop an inflammatory response to the chyle leak; however, their discharge from hospital was delayed. In all those treated conservatively, the chyle leak abated following the introduction of total parenteral nutrition. Furthermore, the use of total parenteral nutrition was not associated with significant morbidity. One patient did however require surgical intervention for a chyle leak. This patient presented late with chylous ascites that failed to respond to conservative management.

Dig Surg 2007;24:418-422 
In summary, compared to the existing literature we have reported a high incidence of chyle leak following surgery for pancreatico-duodenal malignancy. This incidence is partly explained by a policy of early entral nutrition among these patients. Specific attempts at intra-op- erative ligation or peri-operative somatostatin failed to prevent chylous leakage. All those patients who developed a chyle leak remained clinically well and the majority of cases responded quickly to the cessation of oral intake and the introduction of total parenteral nutrition.

\section{References}

1 Browse NL, Wilson NM, Russo F, al-Hassan $\mathrm{H}$, Allen DR: Aetiology and treatment of chylous ascites. Br J Surg 1992;79:11451150

$\checkmark 2$ Morris SA, Taylor SJ: Peripheral nutrition in a case of chyle leak following neck dissection. J Hum Nutr Dietet 2004;17:153-155.

>3 Li S, Zhang Z, Liang X Cui Y: The treatment of chylothorax. Chin Med Sci J 2000;15:253255.

4 Shimizu K, Yoshida J, Nishiura M, Takamochi K, Nakahara R, Nagai K: Treatment strategy for chylothorax after pulmonary resection and lymph node dissection for lung cancer. J Thorac Cardiovasc Surg 2002;124: 499-502.

$\checkmark 5$ Bonavina L, Saino G, Bona D, Abraham M, Peracchia A: Thoracoscopic management of chylothorax complicating oesophagectomy. J Laparoendosc Adv Surg Techniques 2001; 11:367-369.

6 Cheah FC, Noraida MH, Boo NY, Amin TY: Chylothorax after repair of congenital diaphragmatic hernia: a case report. Singapore Med J 2000;41:548-549.

7 Vallieres E, Karmy-Jones R, Wood DE: Early complications. Chylothorax (review). Chest Surg Clin N Am 1999;9:609-616.

$\checkmark 8$ Perez J, Casal J, Rodriguez W: Always remember chylothorax (review). Southern Med J 1999;92:833-835.

$\checkmark 9$ Merigliano S, Molena D, Ruol A, Zaninotto G, Cagol M, Scappin S, Ancona E: Chylothorax complicating oesophagectomy for cancer: a plea for early thoracic duct ligation. J Thorac Cardiovasc Surg 2000;119:453-457.

-10 Fernandez Alvarez JR, Kalache KD, Grauel EL: Management of spontaneous congenital chylothorax: oral medium-chain triglycerides versus total parenteral nutrition. Am J Perinatol 1999;16:415-420.

$\checkmark 11$ Wolthuis A, Landewe RB, Theunissen PH, Westerhuis LW: Chylothorax or leakage of total parenteral nutrition? Eur Resp J 1998; 12:1233-1235.

-12 Nguyen DM, Shum-Tim D, Dobell AR, Techervenkov CI: The management of chylothorax/chylopericardium following paediatric cardiac surgery: a 10-year experience. J Cardiac Surg 1995;10:302-308.
3 Haniuda M, Nishimura H, Kobayashi O, Yamanda T, Miyazawa M, Aoki T, Iida F: Management of chylothorax after pulmonary resection. J Am Coll Surgeons 1995;180 537-540.

14 Milano S, Maroldi R, Vezzoli G, Bozzola G, Battaglia G, Mombelloni G: Chylothorax after blunt chest trauma: an unusual case with a long latent period. Thorac Cardiovasc Surgeon 1994;42:187-190.

15 Terzi A, Furlan G, Magnanelli G, Terrini A, Ivie N: Chylothorax after pleuro-pulmonary surgery: a rare but unavoidable complication. Thorac Cardiovasc Surg 1994;42:81-84

16 Fogli L, Gorini P, Belcastro S: Conservative management of traumatic chylothorax: a case report. Intens Care Med 1993;19:176-177.

17 Marts BC, Naunheim KS, Fiore AC, Pennington DG: Conservative versus surgical management of chylothorax. Am J Surg 1992;164:532-534.

18 Allen EM, Van Heeckeren DW, Spector ML, Blumer JL: Management of nutritional and infectious complications of postoperative chylothorax in children. J Paediatr Surg 1991;26:1169-1174.

19 Grant PW, Brown SW: Traumatic chylothorax: a case report. Aust NZ J Surg 1991;61: 798-800.

20 Cooper P, Paes ML: Bilateral chylothorax. Br J Anaesth 1991;66:387-390.

-21 Brook MP, Dupree DW: Bilateral traumatic chylothorax. Ann Emergency Med 1988;17: 69-72.

22 Leibovitch I, Mor Y, Golomb J, Ramon J: The diagnosis and management of postoperative chylous ascites (review). J Urol 2002;167: 449-457.

23 Hoffman JP, Weese JL, Soli LJ: A single institutional experience with preoperative chemoradiotherapy for stage I-III pancreatic adenocarcinoma. Am Surgeon 1993;59:777-780.

24 Aalami OO, Allen DB, Organ CH: Chylous ascites: a collective review. Surgery 2000; 128:761-778.

25 Haug ES, Saether OD, Odegaard A, Johnsen G, Myhre HO: Chylous complications after abdominal aortic surgery. Int Angiol 1998 17:244-247.

26 Petrasek AJ, Ameli FM: Conservative management of chylous ascites complicating aortic surgery: a case report. Can J Surg 1996;39: 499-501.
27 Geisler JP, Foster RS, Sutton GP: Chyloperitoneum following treatment for advanced gynecologic malignancies. Obstet Gynecol 1994;83:883-885.

$>28$ Bhat AL, Lowery GL: Chylous injury following anterior spinal surgery: case reports. Eur Spine J 1997;6:270-272.

29 Rimensberger PC, Muller-Schenker B, Kalangos A, Beghetti M: Treatment of a persistent postoperative chylothorax with somatostatin. Ann Thorac Surg 1998;66: 253-254

30 Collard JM, Laterre PF, Boemer F, Reynaert M, Ponlot R: Conservative treatment of postsurgical lymphatic leaks with somatostatin14. Chest 2000;117:902-905.

31 Al-Sebeih K, Sadeghi N, Al-Dhahri S: Bilateral chylothorax following neck dissection: a new method of treatment. Ann Otol Rhinol Laryngol 2001;110:381-384

\$2 Ferrandiere M, Hazouard E, Guicheteau V, Gouchet A, Bensenouci M, Lamotte C, Mercier C: Chylous ascites following radical nephrectomy: efficiency of octreotide as treatment of a ruptured thoracic duct. Intens Care Med 2000;26:484-485.

33 Laterre PF, Dugernier T, Reynaert MS: Chylous ascites: diagnosis, causes and treatment (review). Acta Gastroenterol Belg 2000;63: 260-263

34 Spiro JD, Spiro RH, Strag EH: The management of chyle fistulae. Laryngoscope 1990; 100:771-774

35 Reissman P, Teoh TA, Cohen SM, Weiss EG Nogueras JJ, Wexner SD: Is early oral feeding safe after elective colorectal surgery? A prospective randomized trial. Ann Surg 1995; 222:73-77.

36 Stewart BT, Woods RJ, Collopy BT, Fink RJ, Mackay JR, Keck JO: Early feeding after elective open colorectal resections: a prospective randomized trial. Aust NZ J Surg 1998;68: 125-128.

37 Steed HL, Capstick V, Flood C, Schepansky A, Schulz J, Mayes DC: A randomized controlled trial of early versus 'traditional' postoperative oral intake after major abdominal gynecologic surgery. Am J Obstet Gynecol 2002;186:861-865. 